\title{
Pulmonary Microvascular Cytology Can Detect Tumor Cells of Intravascular Lymphoma
}

\author{
Takashi Ishiguro ${ }^{1}$, Noboru Takayanagi ${ }^{1}$, Tsutomu Yanagisawa ${ }^{1}$, Naho Kagiyama ${ }^{1}$, \\ Hiroo Saito ${ }^{1}$, Yutaka Sugita ${ }^{1}$ and Masaru Kojima ${ }^{2}$
}

\begin{abstract}
A 68-year-old man was admitted to our hospital for indistinct consciousness, progressive dyspnea, night sweats and fever of 2 weeks duration. Hypoxemia, thrombocytopenia, and elevated serum lactate dehydrogenase were found. Computed tomography was negative except for a small bilateral pleural effusion. Chest perfusion scintigraphy showed inhomogeneous perfusion thought unlikely to be pulmonary artery thromboembolism. Intravascular large B-cell lymphoma was suspected, and a pulmonary microvascular cytology specimen was obtained that contained numerous large lymphoma cells. Because the patient's condition was rapidly deteriorating, we started chemotherapy on the basis of the pulmonary microvascular cytology findings, and he improved. Later, atypical lymphocytes similar to those in the pulmonary microvascular cytology specimen were found in a bone marrow specimen. He was diagnosed as having diffuse large B-cell lymphoma. Because lymphoma cells were found in the pulmonary microvasculature, intravascular lymphoma was also diagnosed. Pulmonary microvascular cytology was helpful to detect lymphoma cells in the pulmonary microvasculature.
\end{abstract}

Key words: diagnosis, intravascular lymphoma, malignant lymphoma, pulmonary microvascular cytology

(Inter Med 48: 1425-1428, 2009)

(DOI: 10.2169/internalmedicine.48.2338)

\section{Introduction}

Intravascular lymphoma (IVL) is a rare extranodal lymphoma characterized by the presence of tumor cells within blood vessels and is listed as a subtype of diffuse large Bcell lymphoma (1). Because of the lack of lymphadenopathy or mass formation, and due to its rapid and fatal clinical course, antemortem diagnosis of IVL is extremely difficult (2).

The tumor cells are generally present in the small vessels; however, only 5-9\% of patients show peripheral blood involvement (3). The central nervous system, liver, and other organs are often involved (3). Involvement in the lung is not rare (4), and patients with IVL sometimes present with dyspnea as their chief complaint; however, chest X-ray and computed tomography (CT) findings in some patients are normal (3). Several cases have been diagnosed as IVL by transbronchial lung biopsy (TBLB) (5), but severe hypoxemia and thrombocytopenia often make it difficult to perform TBLB.

Here, we present a case of an Asian variant of IVL in a patient presenting with severe thrombocytopenia and hypoxemia. Because the patient's condition did not allow TBLB, we performed pulmonary microvascular cytology (PMC) and detected tumor cells, allowing us to make an early diagnosis of IVL and start chemotherapy that resulted in patient improvement. PMC can be useful to detect tumor cells of IVL especially in patients who cannot tolerate TBLB.

\section{Case Report}

A 68-year-old man was referred to our hospital for somnolence, disorientation, generalized weakness, progressive dyspnea on exertion, night sweats, and fever for 2 weeks.

${ }^{1}$ Department of Respiratory Medicine, Saitama Cardiovascular and Respiratory Center, Kumagaya and ${ }^{2}$ Department of Anatomic and Diagnostic Pathology, Dokkyo Medical University School of Medicine, Tochigi

Received for publication April 5, 2009; Accepted for publication May 8, 2009

Correspondence to Dr. Takashi Ishiguro, k3699001@ pref.saitama.lg.jp 


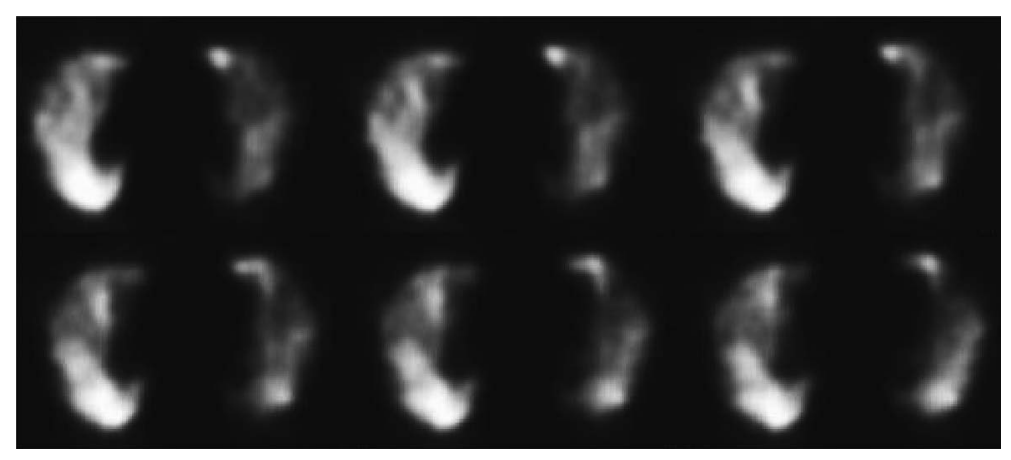

Figure 1. Perfusion scintigraphy. Perfusion scintigraphy showed inhomogeneous perfusion, predominantly in the left lung.

He had been well until this presentation and body weight had been stable. He had never smoked and did not consume alcohol frequently. Ten days before admission, his dyspnea had progressively worsened, and he visited a local physician and was thereafter referred and admitted to the Department of Circulation in our hospital for suspicion of pulmonary artery thromboembolism. On physical examination, the patient showed an increased respiratory rate of 30 breaths per minute without rales, body temperature of $38.3^{\circ} \mathrm{C}$, and no cardiac murmurs. His extremities were edematous, but no eruptions were seen. Blood gas analysis showed a partial pressure of oxygen $\left(\mathrm{PaO}_{2}\right)$ of 64.4 Torr under $5 \mathrm{~L} / \mathrm{min} \mathrm{O}_{2}$ by face mask. Chest X-ray and whole body CT were normal except for a small amount of bilateral pleural effusion and mild hepatosplenomegaly, and no swollen lymph nodes or masses were detected. Laboratory data on admission were as

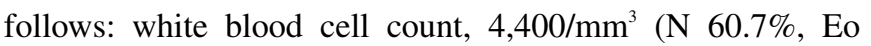
$0.5 \%$, Baso $0.5 \%$, Mono $14.7 \%$, and Ly $23.6 \%$ ); hemoglobin, $14.2 \mathrm{~g} / \mathrm{dL}$; platelet count, $8.2 \times 10^{4} / \mathrm{mm}^{3}$; total protein, $6.4 \mathrm{~g} / \mathrm{dL}$ (albumin, 47.5\%; $\alpha 1,3.1 \% ; \alpha 2,8.2 \% ; \beta ; 9.3 \%$; and $\gamma 31.9 \%$ ); creatinine, $0.8 \mathrm{mg} / \mathrm{dL}$; total bilirubin, $1.3 \mathrm{mg} /$ dL; AST, 108 IU/L; ALT, 30 IU/L; and LDH, 1,166 IU/L. Serum ferritin was elevated at $1,622 \mathrm{ng} / \mathrm{mL}$, as was soluble IL-2 receptor at 1,041 U/mL (normal; 190-650 U/mL). Coagulopathy survey showed the fibrin degradation product level to be slightly elevated at $7.7 \mu \mathrm{g} / \mathrm{mL}$; D-dimer was 4.30 $\mu \mathrm{g} / \mathrm{mL}$. No abnormal cells were detected in a peripheral blood smear. The pleural effusion was too small to obtain a sample by thoracentesis. Perfusion scintigraphy showed inhomogeneous perfusion, predominantly in the left lung (Fig. 1); that we thought it was unlikely related to pulmonary artery thromboembolism. The patient was then transferred to the Department of Respiratory Medicine on day 7 after admission. On the basis of his symptoms, hypoxemia, elevated serum LDH, CT results with absence of swollen lymph nodes and evident masses, and perfusion scintigraphy result, we supposed IVL. After obtaining informed consent, we performed a skin biopsy, bone marrow aspiration cytology, and PMC $(6,7)$, for which a Swan-Ganz catheter was inserted and located in the left pulmonary artery wedge position, as determined by conventional criteria (6), and blood was gently withdrawn from the wedged catheter. Hemody- namic parameters monitored during the procedure included pulmonary capillary wedge, $8 / 6 / 3 \mathrm{mmHg}$; pulmonary artery, $40 / 14 / 23 \mathrm{mmHg}$; and cardiac index, $4.97 \mathrm{~L} / \mathrm{min} / \mathrm{m}^{2}$. No evident complications were noted except for slight bleeding at the puncture site. Extracted blood samples were heparinized and centrifuged. Because malignant cells tend to accumulate in the buffy coat of centrifuged blood (6), slides were made from the buffy coat and were immediately fixed with $95 \%$ alcohol and stained using the Papanicolaou method. The cytological specimens contained several large atypical cells in small, loosely aggregated clusters or in isolation (Fig. 2a). These cells contained narrow amphophilic cytoplasm and had round or slightly indented or irregular nuclei with a thick nuclear membrane and contained one to three small but conspicuous nucleoli and finely dispersed chromatin. Immunochemical analysis showed the tumor cells to be negative for cytokeratin, epithelian membrane antigen, and CD3 and positive for vimentin, CD45, CD79a (Fig. 2b) and CD20. Diffuse large B-cell lymphoma was highly suspected based on these results. Skin biopsy specimen showed no atypical cells, and bone marrow aspiration samples stained with May-Giemsa stain showed hypercellular marrow and an increase in normally shaped megakaryocytes but no atypical cells. After this, hypoxemia and thrombocytopenia were progressively exacerbated, and we started chemotherapy (CHOP therapy: cyclophosphamide, adriamycin, and vincristine, all on day 1, and prednisolone on days 1-5) from day 6 after transfer to the Department of Respiratory Medicine. The patient's $\mathrm{PaO}_{2}$ under ambient air and platelet count returned to normal, and he was discharged. Later, we reevaluated samples of the bone marrow aspirate and found isolated and aggregated large atypical cells similar to those found in the PMC specimen (Fig. 2c) that were L26-positive on immunochemical staining (Fig. 2d). We diagnosed the patient as having intravascular large B-cell lymphoma based on the existence of large atypical lymphocytes in the pulmonary microvasculature and complete absence of swollen lymph nodes and masses, although histological evidence showing filling lymphoma cells in the vessels could not be detected. In addition, hemophagocytosis in the bone marrow, thrombocytopenia, and hepatosplenomegaly, which are characteristic for Asian variant IVL (8), were present; thus the present 

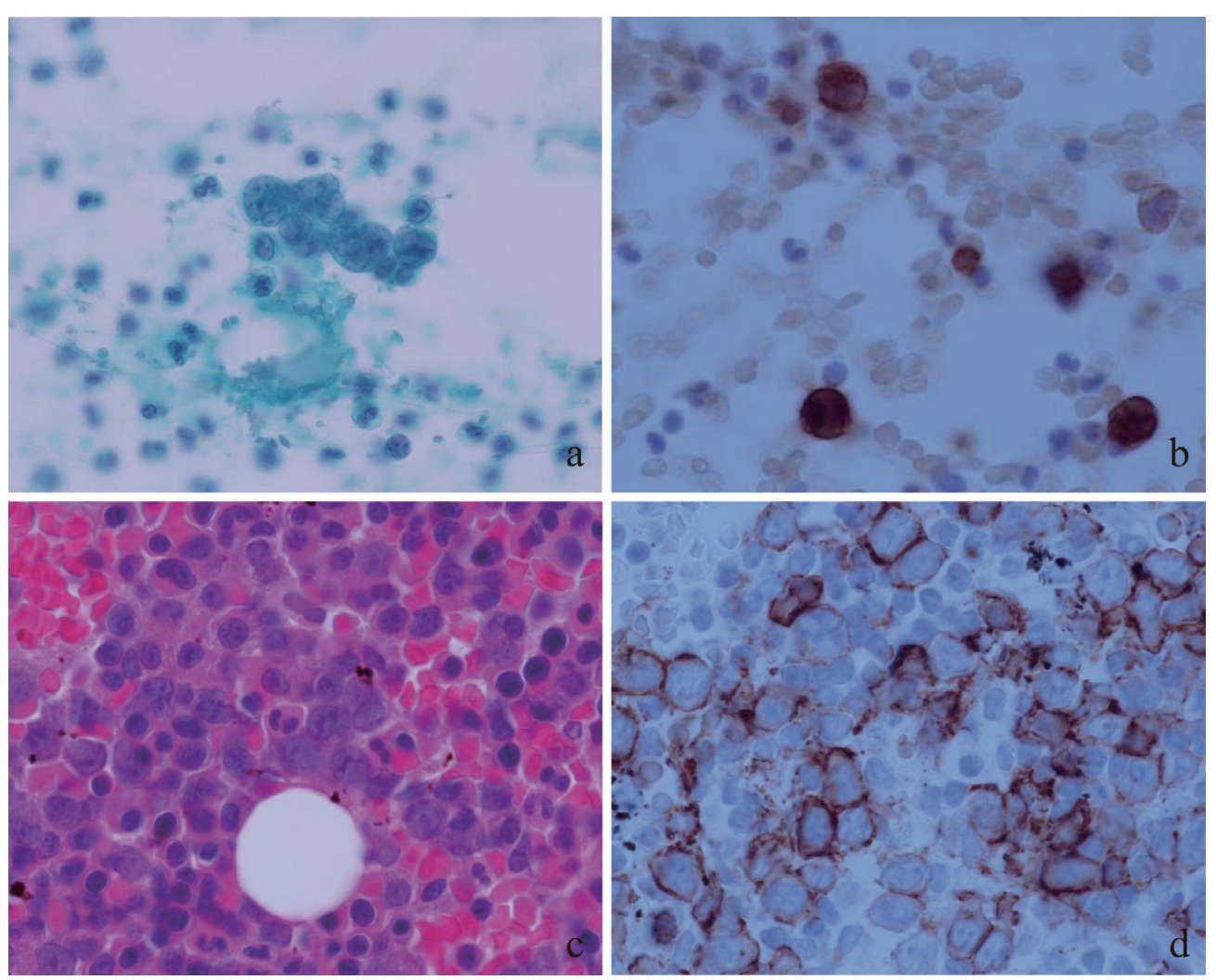

Figure 2. Photomicrographs of pulmonary microvascular cytology and bone marrow aspiration specimens. a) Pulmonary microvascular cytology. Note the loosely aggregated small clusters of large lymphoid cells with narrow amphophilic cytoplasm and round or slightly indented nuclei. Papanicolau stain, $\times \mathbf{1 0 0}$. b) Pulmonary microvascular cytology. Immunochemical staining showed the tumor cells to be CD79a positive. $\times 100$. c) Bone marrow aspiration specimens. Large lymphoid cells are present. Hematoxylin and Eosin staining, $\times 40$. d) Bone marrow aspiration specimens. Immunochemical staining showed the tumor cells to be CD79a positive. $\times 40$.

case was regarded to be an Asian variant of IVL. A second course of chemotherapy was started 4 weeks after the first course, and rituximab was added to the original regimen. The patient has been well without recurrence and will be regularly admitted to hospital to undergo chemotherapy.

\section{Discussion}

We suspected IVL in this patient based on his symptoms, laboratory data, absence of evident swollen lymph nodes or masses, and inhomogeneous pulmonary perfusion. Abnormal lesions were evident in his lungs, and his symptoms indicated central nervous system involvement. Although TBLB should be done in such cases, we considered our patient too ill to tolerate bronchoscopy and TBLB and, and we performed PMC instead, which detected lymphoma cells. Results of immunochemical staining were obtained in only 2 days following blood sampling.

Skin involvement is not uncommon in classical IVL, and skin biopsy sometimes reveals tumor cells within the lumina of the small vessels. However, Asian variants of IVL rarely show skin lesions $(1,4)$, and the skin biopsy of the present patient was normal. In addition, several days are needed to evaluate skin biopsy samples.
PMC, the cytologic study of blood drawn from a wedged pulmonary-artery catheter to detect material in the pulmonary microvasculature was first reported in 1979 in patients with amniotic fluid embolism (9). This procedure has been extended to the diagnosis of fat embolism and pulmonary microvascular metastasis $(6,7,10)$. In 1994, Demirer et al reported that tumor cells of IVL can be detected by PMC (3). They diagnosed IVL in a 58-year-old man with shortness of breath by TBLB, which showed tumor cells within the blood vessels. They also performed PMC, which also detected the tumor cells (3). Abati et al performed PMC in 8 patients with non-Hodgkin's lymphoma who were clinically thought to have pulmonary metastasis (10). PMC results were true positive in 4 , false positive in 1 (pneumonia), false negative in none, and true negative in 2 patients. In the false-positive patient with pneumonia, PMC detected atypical but non diagnostic cells.

We suspected IVL in the present patient but could not perform bronchoscopy and TBLB. We hypothesized that tumor cells should be recognizable in blood extracted from the microvasculature as reported previously (4), and PMC was successful in detecting lymphoma cells in the pulmonary microvasculature of our patient with Asian variant of IVL. Although an invasive procedure, pulmonary artery 
catheterization is generally well tolerated in comparison with TBLB, even in patients with thrombocytopenia and hypoxemia, thus making PMC useful in such cases.

\section{Acknowledgement}

We thank Dr. Akiyoshi Takami (Department of Hematology,
Kanazawa University Hospital), and our collegues (Koichiro Yoneda, Yousuke Miyahara, Daido Tokunaga, Fumiaki Aoki, Kazuyoshi Kurashima, Mikio Ubukata) at Saitama Cardiovascular and Respiratory Center for their detailed comments regarding the present case.

\section{References}

1. Ferreri AJ, Campo E, Seymour JF, et al. Intravascular lymphoma: clinical presentation, natural history, management and prognostic factors in a series of 38 cases, with special emphasis on the "cutaneous variant'. Br J Haematol 127: 173-183, 2004.

2. Harris NL, Jaffe ES, Diebold J, et al. World Health Organization classification of neoplastic diseases of the hematopoietic and lymphoid tissues: report of the Clinical Advisory Committee meetingAirlie House, Virginia, November 1997. J Clin Oncol 17: 38353849, 1999.

3. Demirer T, Dail DH, Aboulafia DM. Four varied cases of intravascular lymphomatosis and a literature review. Cancer 73: 17381745, 1994.

4. Murase T, Nakamura S, Tashiro K, et al. Malignant histiocytosislike B-cell lymphoma, a distinct pathologic variant of intravascular lymphomatosis; a report of five cases and review of the literature. Br J Haematol 99: 656-664, 1997.

5. Kitanaka A, Kubota Y, Imataki O, et al. Intravascular large B-cell lymphoma with FDG accumulation in the lung lacking CT/(67) gallium scintigraphy abnormality. Haematol Oncol 27: 46-49,
2008.

6. Masson RG, Ruggieri J. Pulmonary microvascular cytology a new diagnostic application of the pulmonary artery catheter. Chest $\mathbf{8 8}$ : 908-914, 1985.

7. Masson RG, Krikorian J, Lukl P, Evans GL, McGrath J. Pulmonary microvascular cytology in the diagnosis of lymphangitic carcinomatosis. New Engl J Med 321: 71-76, 1989.

8. Murase T, Nakamura S, Kawauchi K, et al. An Asian variant of intravascular large B-cell lymphoma: clinical, pathological and cytogenetic approaches to diffuse large B-cell lymphoma associated with haemophagocytic syndrome. Br J Haematol 111: 826-834, 2000.

9. Masson RG, Ruggieri J, Siddiqui MM. Amniotic fluid embolism: definitive diagnosis in a survivor. Am Rev Respir Dis 120: 187192, 1979.

10. Abati A, Landucci D, Danner RL, Solomon D. Diagnosis of pulmonary microvascular metastases by cytologic evaluation of pulmonary artery catheter-derived blood specimens. Hum Pathol 25 : 257-262, 1994.

(C) 2009 The Japanese Society of Internal Medicine http://www.naika.or.jp/imindex.html 